\title{
Case Report \\ Mycoplasma pneumoniae as a cause of non-resolving pneumonia in a neonate
}

\author{
Correspondence \\ Surinder Kumar \\ kumarsurinderdr@yahoo.com
}

Received 12 November 2009

Accepted 14 February 2010

\author{
Surinder Kumar, ${ }^{1}$ Arti Maria, ${ }^{2}$ Sanjeev R. Saigal ${ }^{1}$ and Megha Maheshwari ${ }^{1}$ \\ ${ }^{1}$ Department of Microbiology, Maulana Azad Medical College, New Delhi 110002, India \\ ${ }^{2}$ Department of Pediatrics, Maulana Azad Medical College, New Delhi 110002, India
}

\begin{abstract}
Mycoplasma pneumoniae is known to be the chief causative organism for community-acquired non-lobar pneumonia in children of 5-15 years of age. M. pneumoniae as an aetiological agent for pneumonia among neonates and infants has rarely been reported. We report here a case of persistent pneumonia due to M. pneumoniae in a 3-week-old neonate.
\end{abstract}

\section{Introduction}

Approximately 10 to $20 \%$ of all cases of pneumonia are due to Mycoplasma pneumoniae. An increasing incidence of M. pneumoniae infection and disease in children and infants from 0 to 5 years is being reported. The diverse clinical illnesses associated with $M$. pneumoniae infection may lead to a delay in definitive diagnosis.

\section{Case report}

An 18-day-old male neonate born to a 26-year-old secondgravida mother at 39 weeks of gestation was brought in with complaints of fever, cough and coryza for 3-4 days, and excessive crying, respiratory distress and refusal to feed for 1 day prior to admission. There were no significant complaints pertaining to the antenatal or perinatal period. The baby had cried soon after birth and was subsequently exclusively breast fed. At admission, on examination the baby was febrile (temperature $38{ }^{\circ} \mathrm{C}$ ) and hypoxic $(82 \%$ oxygen saturation) with tachypnoea and moderate subcostal-intercostal retractions. Chest examination revealed bilateral equal air entry with scattered crepitations. The results of the rest of the systemic examination were within normal limits. Investigations revealed a total leukocyte count of 27000 cells $\mathrm{mm}^{-3}$; a differential leukocyte count of $47 \%$ polymorphs, $47 \%$ lymphocytes, $1 \%$ monocytes, $5 \%$ eosinophils; an immature to total leukocyte ratio of 0.16 to 1 ; an erythrocyte sedimentation rate of $12 \mathrm{~mm} \mathrm{~h}^{-1}$ in the first hour; and $\mathrm{C}$ reactive protein of $0.24 \mathrm{mg}^{-1}$. Chest X-ray revealed bilateral patchy alveolar opacities suggestive of pneumonia. An X-ray of the neonate during the subsequent course showed consolidation on the right side. Cerebrospinal fluid examination was normal. Blood culture was sent at this time and later found to be sterile.

The baby was presumed to have bacterial pneumonia with probable sepsis and was started on intravenous fluids, oxygen, and intravenous ceftriaxone and amikacin. After 3 days of antibiotic therapy when there was no clinical improvement, as well as the X-ray showing worsening in the form of non-homogeneous opacities in the right upper zone and mid zone with a completely opaque left hemithorax, piperacillin and tazobactam were added. Over the following week the baby continued to be symptomatic and oxygen dependent with moderate respiratory distress, with rates between 70 and 80 breaths $\min ^{-1}$. Additionally, the baby had developed a scattered wheeze with no radiological improvement either. This prompted us to investigate unusual causes of persistent pneumonia. Contrast-enhanced computed tomography of the chest was carried out to rule out any malformation. It revealed confluent air space opacities with air bronchogram in almost the entire left lung and the posterior segment of the right upper lung and apical medial basal segment of right lung suggestive of consolidation in the left lung and posterior segment of right upper and lower lung with minimal left-sided pleural effusion. A dye study, carried out to look for gastro-oesophageal reflux or $\mathrm{H}$ type tracheoesophageal fistula excluded both these conditions. A sweat chloride test was negative ruling out cystic fibrosis. A bronchoscopic examination showed normal results.

Blood cultures for aerobic bacteria and fungal pathogens were found to be negative for growth. A serial urine examination to screen for fungal hyphae gave negative results. Viral studies could not be done due to a lack of facilities. Serology (ELISA; Calbiotech) for IgM and IgG antibodies against Chlamydia pneumoniae were negative on specimens from both the baby and his mother. Paired sera of both the baby and his mother were tested at this time (acute-phase sample) and again after 4 weeks (convalescent sample) for IgM and IgG antibodies against M. pneumoniae by ELISA (NovaTec Immunodiagnostica). Maternal sera tested negative for both maternal IgM and IgG antibodies. However, the baby's sera tested positive and IgM antibodies were found to have a high titre (titre 41.09 in NovaTec units, where a positive result was defined as more than 11 NovaTec units) and IgG antibodies showed rising titres over 4 weeks (IgG titre in acute-phase serum 9.1 NovaTec units, IgG titre in convalescent phase serum 18.4 
NovaTec units, where a positive result was defined as more than 11 NovaTec units). Specimens of nasopharyngeal aspirates and throat-swab specimens were collected for routine aerobic culture on blood and chocolate agar. Culture for M. pneumoniae and PCR to amplify a $543 \mathrm{bp}$ region of the P1 adhesin gene (Williamson et al., 1992) were carried out. The results of both were negative.

Having established the Mycoplasma aetiology of the pneumonia, the baby was started on erythromycin orally [ $40 \mathrm{mg}$ $(\mathrm{kg} \text { body weight })^{-1}$ per day divided into three doses] through a feeding tube as he continued to be symptomatic and oxygen dependent. Other parenteral antibiotics were stopped. Within $4-5$ days of starting erythromycin the baby showed a marked improvement. After 1 week of this therapy the baby no longer needed oxygen, as well as showing radiological clearance of pneumonia. The baby was discharged after completion of a 2 week course of erythromycin.

\section{Discussion}

Respiratory infections remain the most common cause of perinatal morbidity and mortality, despite many advances in neonatal intensive care. $M$. pneumoniae is often the aetiological agent in infections in children older than 5 years and in adolescents (Williamson et al., 1992). M. pneumoniae as an aetiological agent in the causation of neonatal pneumonia has occasionally been reported (Ursi et al., 1995). However, Ureaplasma urealyticum, belonging to the same family (Mollicutes) as M. pneumoniae, is a known cause of congenital pneumonia, especially in preterm neonates (Waites et al., 2005). M. pneumoniae has also been reported to cause congenital pneumonia. We report here an unusual case of non-resolving pneumonia due to $M$. pneumoniae in a 3-week-old neonate. The pneumonia in our case appears to have been acquired postnatally since tests for maternal IgG and IgM antibodies against $M$. pneumoniae were both negative and the baby mounted a twofold rise in antibody titre to M. pneumoniae. Since the mother was IgG negative, it is possible that the baby was susceptible to this infection due to a lack of the protective effect of maternal IgG antibodies against M. pneumoniae.

Our diagnosis was based on serology and definitive therapeutic response to anti-mycoplasma therapy with erythromycin alone. ELISA has been often used as the sole criterion of diagnosis for M. pneumoniae, as PCR and culture may often be negative (Narita, 2005). Although culture for $M$. pneumoniae is the gold standard, it has certain limitations, having little analytical sensitivity even in laboratories where rigorous adherence to procedures known to enhance cultural isolation is used (Waites \& Talkington, 2004). Our negative findings in the Mycoplasma culture tests are, therefore, not surprising. The PCR could have been negative in the present case due to either a low copy number of Mycoplasma cells in the clinical sample, mutation or PCR inhibitors, since our protocol necessarily included both positive and negative controls (Waites et al., 2001). Elevation of M. pneumoniaespecific IgM alone can often be interpreted as evidence of acute infection, since this antibody typically appears within 1 week of initial infection and approximately 2 weeks before IgG antibodies (Sillis, 1990). Moreover, the presence of IgM is considered most significant in the paediatric population, where there has been limited exposure to antigens (Sillis, 1990 ) as observed in the present case where M. pneumoniaespecific IgM antibodies were detected at high titres. Reliable serology is critical for accurate diagnosis of $M$. pneumoniae respiratory disease. The ELISA for the detection of antibody appears to be the method of choice. A recent study (Waites \& Talkington, 2004) conducted to evaluate the utility of ELISA in detecting $\mathrm{IgG} / \mathrm{IgM} / \mathrm{IgA}$ antibodies for diagnosis of $M$. pneumoniae in children has validated the utility of ELISA methodology, in acute phase diagnosis, with a single point serum sample for M. pneumoniae particularly in children. It is not surprising that the IgG rise was only twofold as this could be attributed to the poor antibody response mounted in neonates (Cassell et al., 1996). In spite of broad spectrum antibiotics the baby continued to worsen, with deterioration in both clinical and radiological signs. Although viral studies could not be carried out it seemed unlikely that the clinical and radiological findings befitted a viral aetiology. Serological evidence of M. pneumoniae aetiology, and a good clinical and radiological therapeutic response to erythromycin (a drug specific for $M$. pneumoniae), were sufficient grounds to establish M. pneumoniae aetiology in our case. In conclusion, our key message is that M. pneumoniae should be considered as a probable cause of non-resolving pneumonia in a neonate.

\section{References}

Cassell, G. H., Gambill, G. \& Duffy, L. (1996). ELISA in respiratory infections in humans. In Molecular and Diagnostic Procedures in Mycoplasmology, vol. II, chapter B5, pp. 123-126. Edited by J. G. Tully \& S. Razin. San Diego, CA: Academic Press.

Narita, M. (2005). Evaluation of ELISA kits for detection of Mycoplasma pneumoniae-specific IgG, IgA, IgM antibodies on the diagnosis of Mycoplasma pneumoniae infection in children. Kansenshogaku Zasshi 79, 457-463.

Sillis, M. (1990). The limitations of IgM assay in the serological diagnosis of Mycoplasma pneumoniae infections. J Med Microbiol 33, 253-258.

Ursi, D., Ursi, J.-P., leven, M., Docx, M., Van Reempts, P. \& Pattyn, S. R. (1995). Congenital pneumonia due to Mycoplasma pneumoniae. Arch Dis Child Fetal Neonatal Ed 72, F118-F120.

Waites, K. B. \& Talkington, D. F. (2004). Mycoplasma pneumoniae and its role as a human pathogen. Clin Microbiol Rev 17, 697-728.

Waites, K. B., Bebear, C. M., Robertson, J. A., Talkington, D. F. \& Kenny, G. E. (editors) (2001). Cumitech 34: Laboratory Diagnosis of Mycoplasmal Infections. Washington, DC: American Society for Microbiology.

Waites, K. B., Katz, B. \& Schelonka, R. L. (2005). Mycoplasma and Ureaplasma as neonatal pathogens. Clin Microbiol Rev 18, 757-789.

Williamson, J., Marmion, B. P., Worswick, D. A., Kok, T.-W., Tannock, G., Herd, R. \& Harris, R. J. (1992). Laboratory diagnosis of Mycoplasma pneumoniae infection. 4. Antigen capture and PCR-gene amplification for detection of the mycoplasma: problems of clinical correlation. Epidemiol Infect 109, 519-537. 